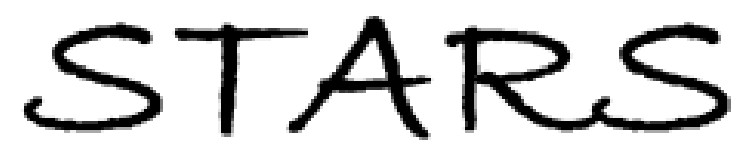

University of Central Florida

STARS

$1-1-2000$

\title{
Systems engineering analysis of aplanatic Wolter type I x-ray telescopes
}

\author{
Patrick L. Thompson \\ James E. Harvey \\ University of Central Florida
}

Find similar works at: https://stars.library.ucf.edu/facultybib2000 University of Central Florida Libraries http://library.ucf.edu

This Article is brought to you for free and open access by the Faculty Bibliography at STARS. It has been accepted for inclusion in Faculty Bibliography 2000 s by an authorized administrator of STARS. For more information, please contactSTARS@ucf.edu.

\section{Recommended Citation}

Thompson, Patrick L. and Harvey, James E., "Systems engineering analysis of aplanatic Wolter type I x-ray telescopes" (2000). Faculty Bibliography 2000s. 2825.

https://stars.library.ucf.edu/facultybib2000/2825

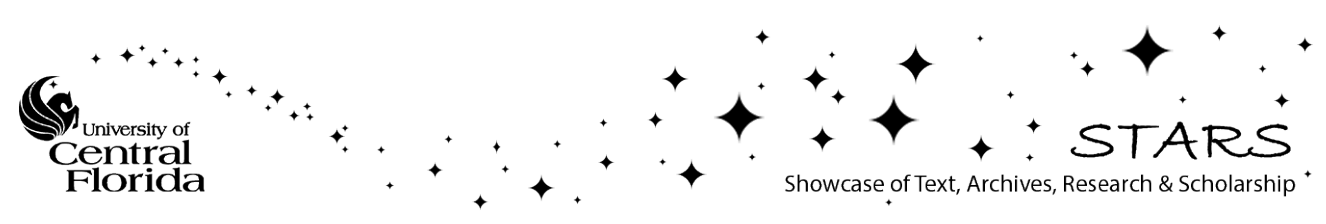




\section{Systems engineering analysis of aplanatic Wolter type I x-ray telescopes}

\author{
Patrick L. Thompson \\ James E. Harvey, FELLOW SPIE \\ University of Central Florida \\ Center for Research and Education in \\ Optics and Lasers (CREOL) \\ P.O. Box 162700 \\ 4000 Central Florida Blvd. \\ Orlando, Florida 32816 \\ E-mail: harvey@creol.ucf.edu
}

\begin{abstract}
It is well known that normal-incidence aplanatic telescope designs perform better at small field angles than ones corrected only for spherical aberration. This is why most large astronomical telescopes fabricated in the past fifty years have been of the Ritchey-Chretien (aplanatic) design rather than of the classical Cassegrain design. For the relatively new field of x-ray astronomy, the Wolter type I grazing incidence design has been extensively utilized. It consists of a paraboloidal primary mirror coaxial with a confocal hyperboloidal secondary mirror. Aplanatic versions of the Wolter type I grazing incidence $x$-ray telescope have been discussed in detail in the literature, and are widely touted as being superior designs. However, scattering effects from residual optical fabrication errors and other practical engineering error sources prevent these grazing-incidence telescopes from being near diffraction-limited (even on axis) at the very short operational x-ray wavelengths. A systems engineering analysis of these error sources indicates that they will dominate coma at the small field angles, and of course astigmatism, field curvature, and higher-order aberrations dominate coma at the large field angles. Hence, there is little improvement in performance when going to an aplanatic design. Comparison of performance predictions for the classical versus aplanatic Wolter type I $\mathrm{x}$-ray telescope are presented for the special case of the Solar X-Ray Imager (SXI) baseline design. SXI is expected to become a standard subsystem aboard the next generation of NOAA/GOES weather satellites. (c) 2000 Society of Photo-Optical Instrumentation Engineers. [S0091-3286(00)01606-8]
\end{abstract}

Subject terms: aplanatic; grazing incidence; Wolter type I; x-ray telescopes; SXI; GOES.

Paper 990204 received May 24, 1999; revised manuscript received Nov. 17, 1999; accepted for publication Nov. 30, 1999.

\section{Historical Background}

In 1672 a French sculptor by the name of Cassegrain suggested the use of a convex hyperboloidal mirror as secondary to a confocal primary paraboloid in order to control aberrations in reflective astronomical telescopes. This leap in optical design owed itself chiefly to the observed opposing effects of the concave primary and convex secondary mirrors with respect to off-axis aberrations. Up until that time, only Gregorian telescope designs (which incorporated concave elliptical secondary mirrors) were considered superior to simple paraboloids, and they were also known to be unmakable. Moreover, Sir Isaac Newton himself passionately argued to the academy that such designs ought not even to be considered, due to their impractical and unobtainable surface figures that would result in more severe aberrations than his own (simpler) design if they were not made absolutely perfect. Indeed, the Newtonian telescope became the cornerstone of practical observational astronomy for many years. It was not until about 1780, when optical instrument makers rediscovered the superior design of Cassegrain, that the concept was taken seriously at all. Unfortunately, it took altogether nearly 200 years after Cassegrain's suggestion for engineers and technicians to mas- ter the fabrication and alignment of multiple aspherical surfaces necessary for the construction of a practical Cassegrain telescope. ${ }^{1}$

In the mid 1850s, Ludwig Von Seidel formulated a thorough and comprehensive mathematical treatment of the (previously observed) aberrations inherent in refractive and reflective optical imaging systems. Armed with his knowledge of aberration theory, George W. Ritchey, an American telescope maker, together with the French optician Henry Chretien, devised an entirely new kind of telescope in the 1920s. This aplanatic Cassegrain design was first built by Ritchey in 1930 as the 40-in. Ritchey-Chretien telescope at the U.S. Naval Observatory in Washington. ${ }^{1-3}$

Overlapping the previously described events at the turn of the twentieth century were the discovery, science, and eventual technology of x-rays. Roentgen's serendipitous discovery of this new radiation in 1895 led to Von Laue's famous demonstration of $\mathrm{x}$-ray diffraction through thick crystals in 1912. Compton eventually proved the electromagnetic nature of x-rays in 1923 by reflecting them from highly polished metallic surfaces at grazing incidence $(\lessgtr 1$ deg). This led to the inescapable conclusion that the index of refraction of metals at X-ray wavelengths must be less than unity, by way of total external reflection. In fact, the optical index of most materials is highly complex for $\mathrm{x}$ 
rays. By 1929, Jentzsch argued that it was virtually impossible to form good images with $\mathrm{x}$ rays through use of a single mirror, so he proposed the application of two toroidal or cylindrical mirrors with differing curvatures. Coexisting with all this geometrical difficulty of imaging $\mathrm{x}$ rays were the ubiquitous effects of grazing incidence surface scattering, first analyzed thoroughly by Ehrenberg in 1949. Ehrenberg demonstrated that surface roughness could be represented by Fourier series decomposition, and thus, he semirigorously predicted (through the grating equation and scalar diffraction theory) the effects of scattering on image quality. ${ }^{4}$

Still, these primitive optical systems were only onedimensional "imagers" with small collecting areas and extreme scattering. The first two-dimensional image produced by controlled $\mathrm{x}$-ray deflection was obtained by Kirkpatrick and Baez in 1948. ${ }^{5}$ In order to get 2-D imaging with large collecting area, a German physicist by the name of Wolter ${ }^{6}$ (1952) designed a set of coaxial cone-shaped grazing incidence mirrors, each with an aspheric (parabolic, hyperbolic, or elliptic) departure imprinted on its surface. These twomirror systems (Wolter types I, II, and III) were axially symmetric, were confocal, and followed the principles of on-axis stigmatic imaging laid down over 300 years ago by Newton, Gregory, and Cassegrain. Wolter did not attempt to optimize the first designs for off-axis or finite conjugate imaging; however, he did write a second paper that same year attempting to formulate completely aplanatic versions of his designs (the so-called Wolter-Schwarzschild designs). ${ }^{7}$ Although his aim was to create an X-ray microscope, Wolter unwittingly became the father of modern $\mathrm{x}$-ray astronomy eleven years later, when on October 15, 1963 the first Wolter-type x-ray telescope was launched into space. ${ }^{4}$

Over the past thirty years, many attempts have been made at fully understanding, optimizing, and improving on the original Wolter designs, but they have only met with limited success. One attempt was to avoid the problems of grazing incidence altogether by depositing multiple highand low-atomic-number thin films onto more conventional Schwarzschild configurations. However, this has proven effective only for wavelengths longer than about $40 \AA$, and then only for very narrow bandwidths. In the years 1972 and 1973, Van Speybroeck and Chase began taking advantage of the latest computerized ray-tracing algorithms to determine empirically and parametrically the effects of varying designs on the imaging performance of the Wolter ${ }^{8}$ and Wolter-Schwarzschild ${ }^{9}$ type I telescopes. Their findings were extremely useful, but apparently lacked the identification and interpretation of aberrations in the normal sense (defocus, spherical aberration, coma, astigmatism, field curvature, distortion, etc.). In 1977, Werner ${ }^{10}$ attempted the computational optimization of a Wolter type I telescope by relaxing the surface shape constraint to that of a generalized axial polynomial. This resulted in almost flat imaging response across the field of view, but simultaneously sacrificed the possibility of diffraction-limited performance. The year 1977 also brought Winkler and Korsch $^{11}$ to attention through their apparently decisive and thorough formulation of two-mirror grazing incidence aberration theory. The results appeared to show, however, (due to their limited precision) that any classical Wolter- type telescope was already aplanatic! Even Wolter himself did not agree with this, as evidenced by his second paper. ${ }^{7}$ The year 1979 saw the arrival of a paper by Cash et al. ${ }^{12}$ concluding that standard, near-normal-incidence aberration theory could be applied exactly to grazing incidence optical elements. Korsch ${ }^{13}$ showed (even with his low precision) that there exists a first-order coma term not present in normal aberration theory for a single mirror. Also, Nariai ${ }^{14}$ stated quite decisively in 1987 that "it is not possible to use ordinary aberration theory because the expansion of aberrations in series of powers on the height of the object and on the radius of the pupil does not converge,...." Besides this, Nariai ${ }^{15}$ also showed analytically in 1988 that all aberrations in his own expansion must be integrated over the entire annular pupil. Aberration coefficients in grazing incidence systems are apparently themselves a function of pupil coordinates. In 1985, Saha $^{16}$ discussed the transverse ray aberrations of paraboloid-hyperboloid telescopes, and in $1986,,^{17} 1987,{ }^{18}$ and $1988^{19}$ he performed an extensive analysis of the aberrations of all types of Wolter grazing incidence and all combinations of normal-incidence paraboloid-hyperboloid and paraboloid-ellipsoid telescopes.

When all is said and done on the subject of grazing incidence $\mathrm{x}$-ray telescope designs, eventually the telescope has to be fabricated, assembled, aligned, mounted on an appropriate platform, and probably launched for spacebased observations. To this end, we will show (in analogy with Newton's argument on practicality) that current stateof-the-art optical fabrication, metrology, and alignment errors dominate the effects of coma for small field angles; and of course, astigmatism dominates coma for large field angles. Hence, there is little improvement in performance to be gained from the "aplanatic" (coma-corrected) design. Indeed, we will show that there is no more than $10 \%$ improvement in performance of an "aplanatic" design over that of the classical Wolter type I for the special case of the Solar X-ray Imager (SXI) baseline design. SXI is a complimentary, add-on instrument designed primarily for use on the GOES next-generation geosynchronous weather satellites. However, its modular design is suitable for installation on many other spacecraft platforms. Its primary mission is to continuously observe the full solar disk, including coronal holes, active regions, flares, and coronal mass ejections. $^{20}$

\section{The Aplanatic Cassegrain Telescope}

The classical Cassegrain telescope exhibits a perfect geometric image on axis because it is corrected for all orders of spherical aberration. However, the design is over constrained in that the paraboloidal primary mirror and the hyperboloidal secondary mirror are individually corrected for spherical aberration. On requiring only that the twomirror system be corrected for spherical aberration, there are sufficient degrees of freedom to achieve the aplanatic (zero spherical aberration and coma) Ritchey-Chretien twomirror telescope with a hyperboloid-hyperboloid design. Both designs suffer from field curvature and astigmatism.

Figure 1 illustrates the geometrical rms image radius (obtained from real ray trace data) plotted as a function of field angle for both a classical Cassegrain and the RitcheyChretien telescope design with the diameter $(D$ 


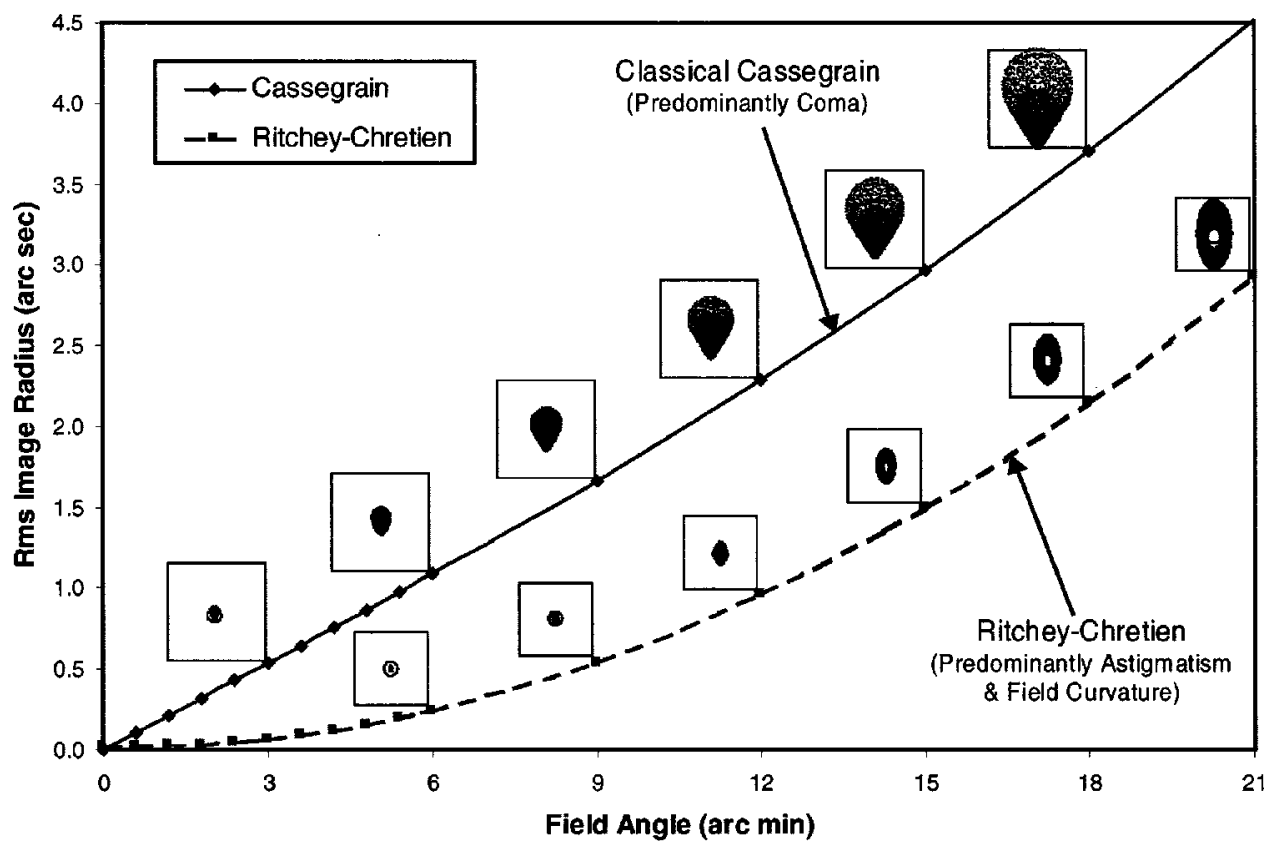

Fig. $1 \mathrm{Rms}$ image radius plotted as a function of field angle for both a classical Cassegrain and its aplanatic counterpart, the Ritchey-Chretien telescope. Geometrical spot diagrams indicating residual aberrations are also illustrated.

$=160 \mathrm{~mm})$ and effective focal length $(f=655 \mathrm{~mm})$ of the SXI Wolter type I baseline design. The 21-arcmin half field angle also comes from the SXI requirements. The fact that coma varies linearly with field angle, while field curvature and astigmatism vary quadratically with field angle, are evident from these curves. The zero slope of the curve for the aplanatic Ritchey-Chretien design at small field angles indicates an absence of coma. Geometrical spot diagrams dramatically indicate that coma dominates the performance of the classical Cassegrain design, whereas the RitcheyChretien design exhibits only field curvature and astigmatism.

The improvement in geometrical performance is very substantial when going from a classical Cassegrain to the aplanatic Ritchey-Chretien telescope design. Figure 2 shows the percentage reduction in geometrical rms image size as a function of field angle for several different telescope focal ratios.

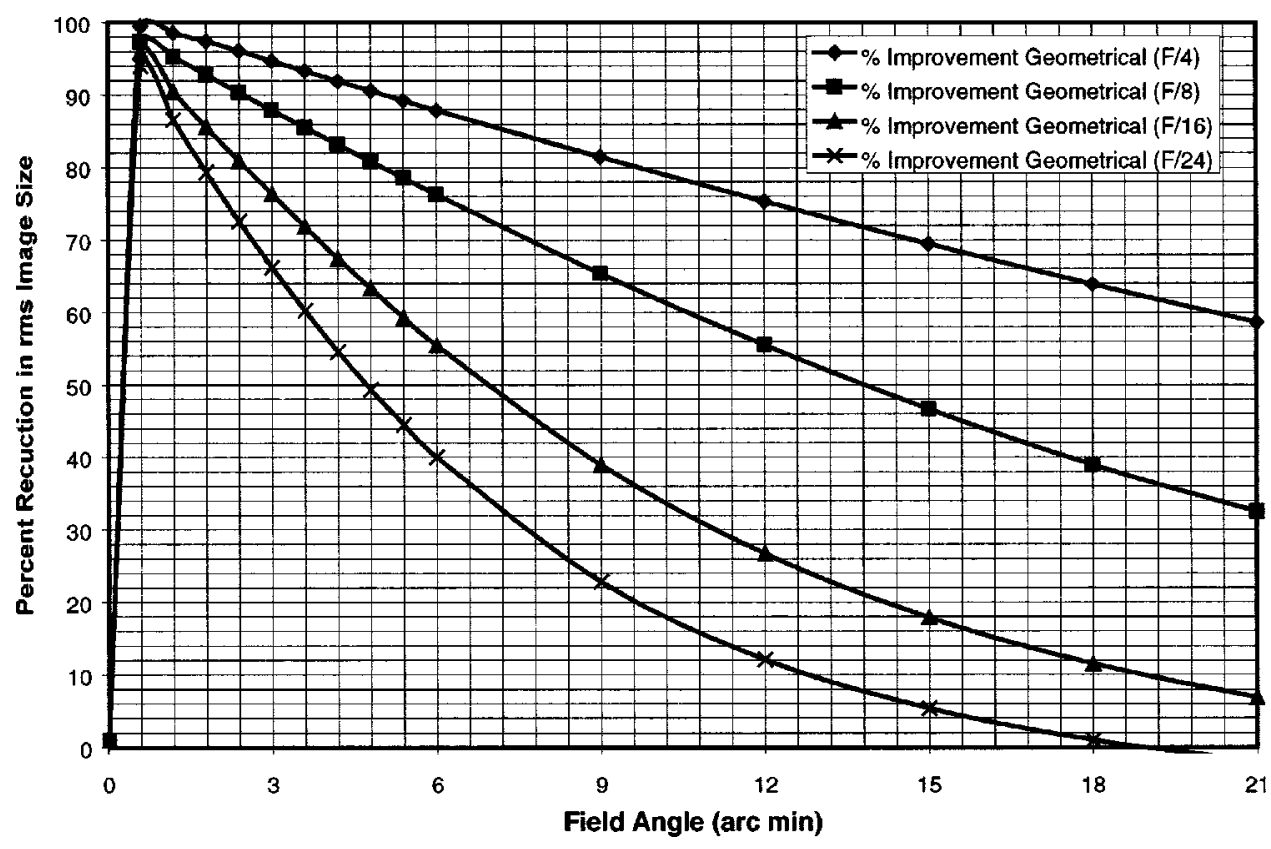

Fig. 2 Percentage reduction in rms image radius achieved with aplanatic designs, plotted as a function of field angle for Cassegrain telescopes of different focal ratios. 


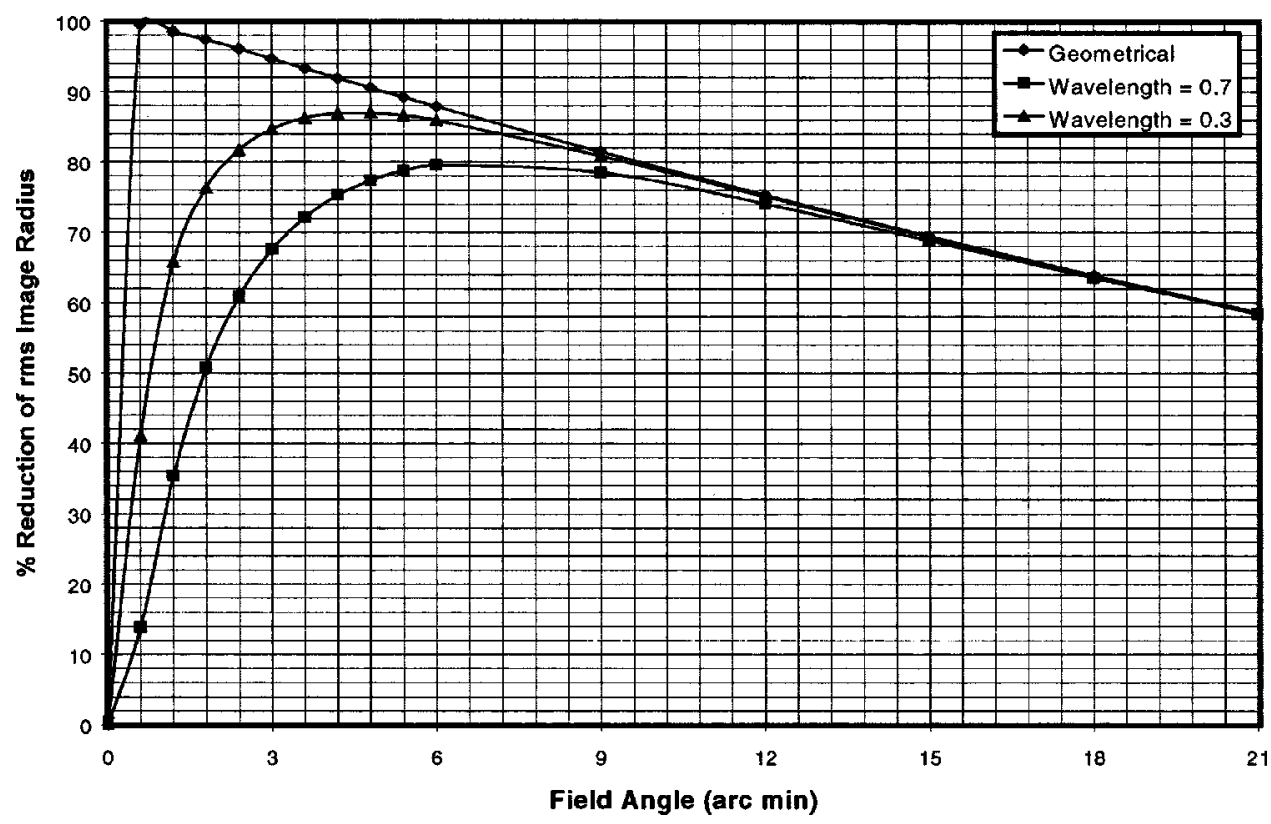

Fig. 3 Percentage reduction in rms image radius due to the aplanatic design when considering geometrical aberrations and diffraction effects at visible wavelengths.

Note that the improvement is greatest (almost 100\% reduction in rms image size) for very small field angles and falls off with increasing field angle as the contribution from astigmatism and field curvature increases relative to coma for the Cassegrain design. This falloff in performance improvement occurs much more rapidly for large focal ratios.

Conventional normal-incidence, two-mirror telescopes can be fabricated, assembled, and aligned to exhibit neardiffraction-limited performance on axis at visible wavelengths. The actual point spread function (PSF) of an imaging system degraded by diffraction and geometrical aberrations can be approximated by the convolution of the diffraction-limited PSF and the geometrical PSF (spot diagram). The rms radius of the resulting image is just the root-sum-square (rss) of the geometrical rms radius and the rms radius of the diffraction-limited PSF. ${ }^{21}$ Figure 3 thus plots the reduction in rms image radius as a function of field angle for geometrical design errors only, and when including diffraction effects for two different wavelengths when going from an $f / 4$ classical Cassegrain design to the aplanatic Ritchey-Chretien design. Even with diffraction effects included, the optical performance of the aplanatic Ritchey-Chretien telescope is far superior to that of the classical Cassegrain telescope. This is why most large astronomical telescopes fabricated in the past fifty years have been of the Ritchey-Chretien (aplanatic) design rather than of the classical Cassegrain design.

It is this well-known increase in performance of conventional normal-incidence two-mirror telescopes operating at visible wavelengths that has led to the assumption that a similar improvement can be achieved by going to an aplanatic version of the classical Wolter type I grazing incidence $\mathrm{x}$-ray telescope.

\section{Fundamentals of Wolter Type I Telescopes}

A Wolter type I grazing incidence $\mathrm{x}$-ray telescope made up of a paraboloid and hyperboloid is illustrated in Fig. 4. The equation for a paraboloid with its vertex at $z_{p}$ is given by $r_{p}^{2}=2 R_{p}\left(z-z_{p}\right)$,

where $R_{p}$ is the paraboloid vertex radius of curvature and $r_{p}$ is the radius of the paraboloid at the axial position $z$. The equation for a hyperboloid centered at $z_{h}$ is given by

$\frac{\left(z-z_{h}\right)^{2}}{a^{2}}-\frac{r_{h}^{2}}{b^{2}}=1$

where $a$ and $b$ are the semimajor and semiminor axes of the hyperboloid. The eccentricity of the hyperboloid is determined by $a$ and $b$ :

$\epsilon=\left(\frac{b^{2}}{a^{2}}+1\right)^{1 / 2}$

The separation of the two hyperboloid foci is given by $2 a \epsilon$. If we superpose the rear hyperboloid focus on the paraboloid focus, the front hyperboloid focus becomes the system focus and $f^{\prime}=z_{f}-z_{0}$ becomes the nominal focal length of the telescope. This is accomplished by positioning the origin of our coordinate system an arbitrary distance $z_{1}$ in front of the front edge of the paraboloid mirror and setting

$z_{p}=z_{1}+L_{p}+\frac{\text { gap }}{2}+f^{\prime}+2 a \epsilon+\frac{R_{p}}{2}$

and

$z_{h}=z_{1}+L_{p}+\frac{\text { gap }}{2}+f^{\prime}+a \epsilon$, 


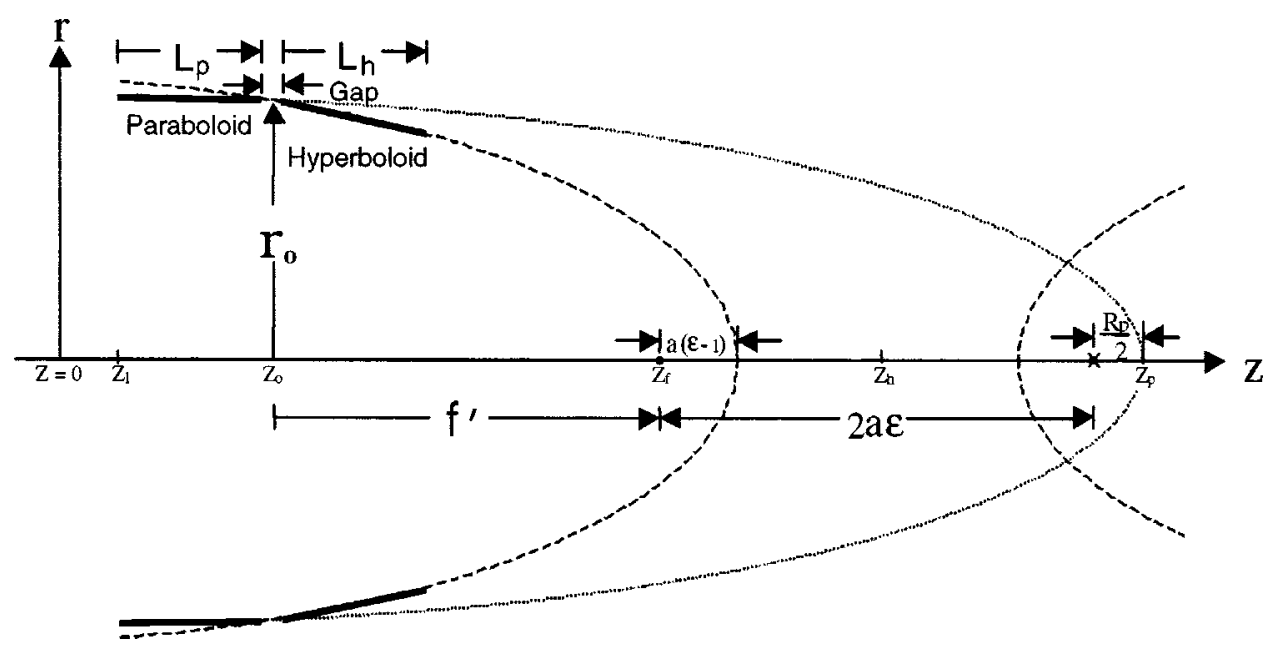

Fig. 4 Wolter type I grazing-incidence telescope configuration.

where $L_{p}$ is the length of the paraboloid mirror and gap is the width of the gap between the paraboloid and the hyperboloid.

The optical prescription of a classical Wolter type I $\mathrm{x}$-ray telescope can thus be completely specified by the three independent parameters $R_{p}, a$, and $b$ (or $R_{p}, a$, and $\epsilon)$. An optimized (maximized effective collecting area) Wolter type I telescope can be obtained if we require the grazing angles of reflection from the paraboloid and the hyperboloid to be equal near their point of intersection. This constraint reduces the number of independent parameters in the optical prescription to two.

For our purposes it is more convenient to choose the telescope radius at the intersection of the paraboloid and the hyperboloid, $r_{0}$, and the nominal focal length of the telescope, $f^{\prime}$, as the parameters of the optical prescription. The grazing angle at the joint is then given by

$\alpha=\frac{1}{4} \arctan \left(\frac{r_{0}}{f^{\prime}}\right)$.

The actual focal length, as measured from the system principal (nodal) point, is slightly larger than the nominal focal length:

$f=f^{\prime}+\frac{r_{0}^{2}}{2 f^{\prime}}$,

and the plate scale is the reciprocal of this focal length, expressed in arcseconds per micrometer.

In addition to the telescope radius $r_{0}$ and the nominal focal length $f^{\prime}$, the optical design parameters include the length of the paraboloid mirror, $L_{p}$, the length of the hyperboloid mirror, $L_{h}$, and the width of the gap between the two mirror elements. From these input parameters, the actual dimensions of the mirror elements can be calculated as well as the obscuration ratio of the collecting aperture, which determines both the geometrical collecting area and the diffraction-limited image characteristics.
The SXI baseline design system parameters, optical prescription, and other significant quantities are tabulated in Table 1.

\subsection{Geometrical Performance of the Classical Wolter Type I X-ray Telescope}

The classical Wolter type I x-ray telescope design produces an ideal on-axis geometrical point image (zero spherical aberration); however, field curvature is a dominant limiting factor determining the off-axis performance of grazing incidence $\mathrm{x}$-ray telescopes if a flat detector or focal plane must be used. The severe field curvature of the above SXI baseline design is illustrated in Fig. 5 by plotting the optimum focal surface and comparing it with that of a classical Cassegrain with the same focal ratio. The axial focal position was determined by minimizing the geometrical rootmean-square (rms) image size. The focal plane of such systems is frequently despaced to improve the off-axis performance, although this results in a degraded (defocused) on-axis image.

Geometrical-optical performance as determined from ray-trace data is conveniently expressed in terms of rms image radius in arcseconds. This quantity is calculated and plotted as a function of field angle for several different axial positions of the focal plane in Fig. 6. Also shown for comparison is the performance curve that would be achieved with a curved detector conforming to the optimally curved focal surface.

Note that the curve for the best focal surface in Fig. 6 appears to have a linear and a quadratic component. This is consistent with findings of Van Speybroeck and Chase. ${ }^{8}$ For small field angles, the linear component dominates and will be associated with a conventional comalike aberration. $^{22,23}$ Similarly, the quadratic component of the curve will be associated with a conventional astigmatism like aberration. ${ }^{22,23}$ The curve corresponding to the Gaussian image plane also appears to consist primarily of a linear and a quadratic component. The linear component (coma) is same as for the best focal surface, as evidenced by the slope at small field angles. However, the quadratic component is significantly larger, since it contains contributions 
Thompson and Harvey: Systems engineering analysis ...

Table 1 Various SXI baseline geometrical and optical parameters.

\begin{tabular}{|c|c|c|c|}
\hline \multirow[b]{2}{*}{ Parameter } & \multicolumn{3}{|c|}{ Value } \\
\hline & $\begin{array}{l}\text { Telescope } \\
\text { system }\end{array}$ & $\begin{array}{l}\text { Paraboloid } \\
\qquad(m=\mathrm{p})\end{array}$ & $\begin{array}{c}\text { Hyperboloid } \\
(m=h)\end{array}$ \\
\hline Joint focal length $f^{\prime}(\mathrm{mm})$ & 655 & - & - \\
\hline Radius of joint, $r_{0}(\mathrm{~mm})$ & 80 & - & - \\
\hline Optic length $L_{m}(\mathrm{~mm})$ & - & 47.5 & 47.5 \\
\hline Gap about joint, $g(\mathrm{~mm})$ & 5 & - & - \\
\hline Grazing angle at joint, $\theta_{i}(\mathrm{deg})$ & 1.74086713 & - & - \\
\hline Nodal focal length $f(\mathrm{~mm})$ & 659.88549618 & - & - \\
\hline Plate scale $m(\operatorname{arcsec} / \mu \mathrm{m})$ & 0.31257666 & - & - \\
\hline Vertex radius $R_{v m}(\mathrm{~mm})$ & - & -2.43145733 & -2.44046651 \\
\hline Semimajor axis a $(\mathrm{mm})$ & - & - & 328.71572867 \\
\hline Semiminor axis $b(\mathrm{~mm})$ & - & - & 28.32348367 \\
\hline Eccentricity $\epsilon$ & - & 1.00000000 & 1.00370526 \\
\hline Conic constant $-\epsilon^{2}$ & - & -1.00000000 & -1.00742425 \\
\hline Separation of foci, $2 a \epsilon(\mathrm{mm})$ & - & - & 659.86741092 \\
\hline Separation of vertices, $z_{p h}(\mathrm{~mm})$ & 659.86516279 & - & - \\
\hline Inner radius $r_{m \min }(\mathrm{mm})$ & - & 80.07594699 & 75.41433272 \\
\hline Midplane radius $r_{m \text { mid }}(\mathrm{mm})$ & - & 80.79388287 & 77.59649010 \\
\hline Outer radius $r_{\max }(\mathrm{mm})$ & - & 81.50549511 & 79.77145135 \\
\hline Front position $z_{\text {mfront }}(\mathrm{mm})$ & - & 0.00000000 & 52.50000000 \\
\hline Midplane position $z_{m m i d}(\mathrm{~mm})$ & - & 23.75000000 & 76.25000000 \\
\hline Rear position $z_{\text {mrear }}(\mathrm{mm})$ & - & 47.50000000 & 100.00000000 \\
\hline Linear obscuration ratio & 0.98246071 & - & - \\
\hline Geometrical collecting area $A\left(\mathrm{~mm}^{2}\right)$ & 725.67160688 & - & - \\
\hline Location of focus, $z_{\text {mfoc }}(\mathrm{mm})$ & - & 1374.86741092 & 705.00000000 \\
\hline
\end{tabular}

from both astigmatism and field curvature. Without attempting to develop any rigorous aberration theory, we will, throughout this paper, refer to the image degradation (indicated by rms image size) that is linear in field angle as coma, and to the degradation that is quadratic in field angle as a combination of astigmatism and field curvature. Similarly, we will consider any image degradation on-axis to be caused by a combination of defocus and spherical aberra-

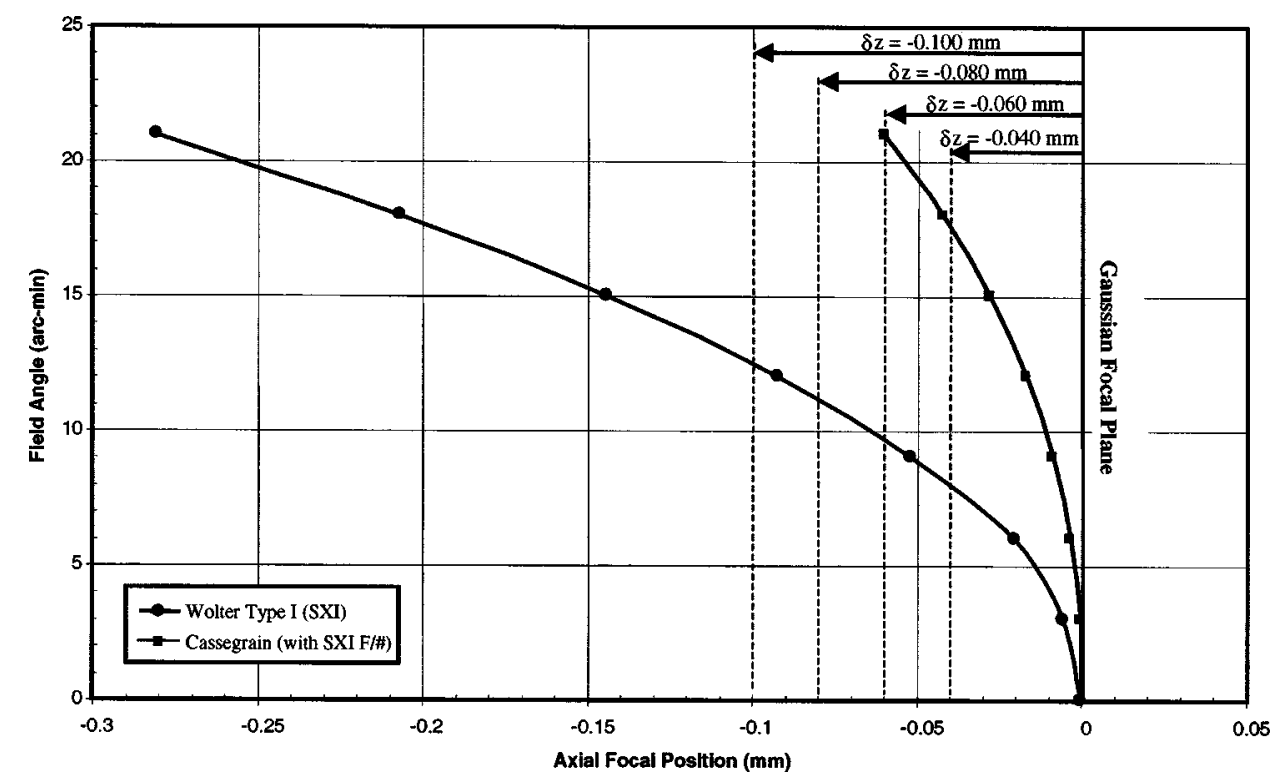

Fig. 5 Field curvature exhibited by the SXI baseline design compared with the field curvature of a normal incidence Cassegrain telescope with the same focal ratio. 


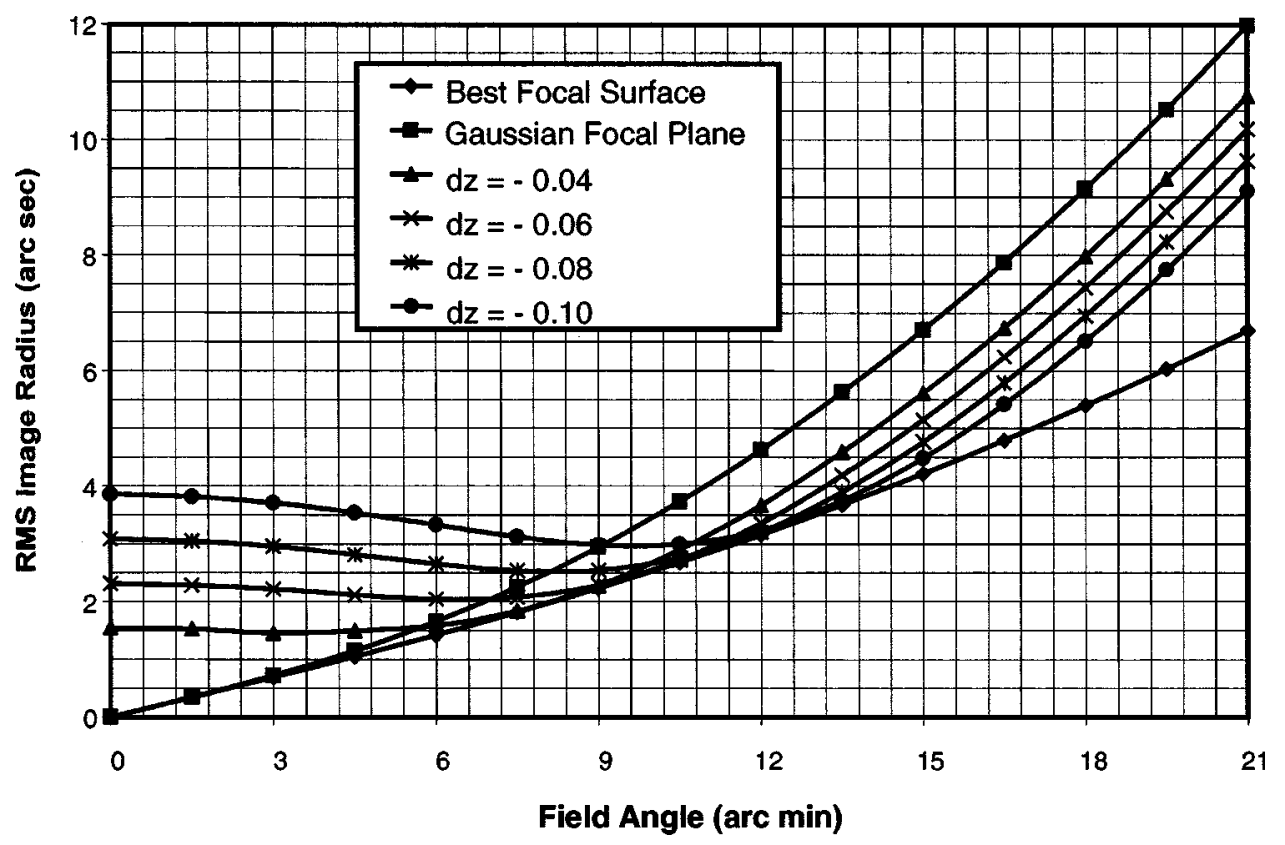

Fig. 6 Geometrical performance of a classical Wolter type I telescope design for different axial positions of the focal plane.

tion. Clearly, we are not trying to distinguish between various orders of aberrations. For example, the linear component of these curves includes all orders of linear coma. Likewise, the quadratic component includes third-order field curvature and astigmatism as well as all higher-order aberration terms that have a quadratic dependence on field angle; this includes the fifth-order aberration usually referred to as oblique spherical aberration. ${ }^{10,14-19}$ There are also, no doubt, cubic and higher-order contributions to the curves in Fig. 6; however, they do not appear to play a significant role for field angles less than 21 arcmin.

\subsection{Geometrical Performance of an Aplanatic Wolter Type I X-ray Telescope}

An aplanatic design corresponding to the above classical Wolter type I x-ray telescope design can be determined empirically by allowing the vertex radii, mirror conic constants, and vertex-to-vertex separation to vary while utilizing the numerical optimization capability of a state-of-theart optical design code. Care must be taken to construct a merit function that adequately constrains the first-order optical properties of the telescope. In our case, we made extensive but careful use of the program ZEMAX. ${ }^{24}$ Although ZEMAX exhibits several inherent difficulties associated with grazing-incidence systems, the basic ray tracing features were shown to be consistent with results computed by the optical surface analysis code (OSAC) which was developed specifically for the analysis of grazing-incidence $\mathrm{X}$-ray telescopes. ${ }^{25}$ OSAC does not have an optimization capability.

The ZEMAX parameters of the SXI baseline design are presented in Table 2 according to the standard lens-editor format for the program. Note that the conic constants listed are equal to $-\epsilon^{2}$, and that the conic-constant magnitude for the primary mirror (surface 5) is unity. This design is thus a classical Wolter type I X-ray telescope (paraboloidhyperboloid). Also shown in Table 2 is an optimal spherical focal surface (surface 8 ), which has a radius of about -35 $\mathrm{mm}$. This allows us to evaluate the geometrical performance on both a plane and a curved focal surface. The

Table 2 ZEMAX lens-editor values for SXI baseline.

\begin{tabular}{rlccccc}
\hline \hline Surface & Type & Radius & Thickness & Glass & Semidiameter & Conic \\
\hline OBJ & Standard & Infinity & Infinity & - & Infinity & 0 \\
$1^{*}$ & Standard & Infinity & 75 & - & 81.55131000 & 0 \\
2 & Standard & Infinity & 50 & - & 100 & 0 \\
3 & Standard & Infinity & 50 & - & 100 & 0 \\
4 & Standard & Infinity & 1266.08313959 & - & 100 & 0 \\
STO* & Standard & -2.43145733 & -659.86516279 & Mirror & 81.50549511 & -1.00000000 \\
$6^{*}$ & Alternate & -2.44046651 & -1.21797680 & Mirror & 80 & -1.00742425 \\
7 & Standard & Infinity & 0 & - & 10 & 0 \\
IMA & Alternate & -35.00 & - & - & 10 & 0 \\
\hline \hline
\end{tabular}


Thompson and Harvey: Systems engineering analysis ...

Table 3 ZEMAX lens-editor values for aplanatic SXI.

\begin{tabular}{rcccccc}
\hline \hline Surface & Type & Radius & Thickness & Glass & Semidiameter & Conic \\
\hline OBJ & Standard & Infinity & Infinity & - & Infinity & 0 \\
$1^{*}$ & Standard & Infinity & 75 & - & 81.55309490 & 0 \\
2 & Standard & Infinity & 50 & - & 100 & 0 \\
3 & Standard & Infinity & 50 & - & 100 & 0 \\
4 & Standard & Infinity & 1306.9819203 & - & 100 & 0 \\
STO* & Standard & -2.28470537 & -709.9089603 & Mirror & 81.50728000 & -1.00010828 \\
$6^{*}$ & Alternate & -2.57809467 & 7.927040121 & Mirror & 80 & -1.00731680 \\
7 & Standard & Infinity & 0 & - & 10 & 0 \\
IMA & Alternate & -35.00 & - & - & 10 & 0 \\
\hline \hline
\end{tabular}

purpose of surfaces 1 through 4 and 7 is simply to provide mechanical reference planes from which to track the relative positions of surface limits and rays within the layout.

Table 3 represents a coma-corrected version of the baseline design, where the first-order properties (joint radius, nominal joint focal length, and shell lengths) have been forced to remain invariant. Only the vertex curvatures, conic constants, and thicknesses were allowed to vary during the optimization process. The optimization was performed by trying to simultaneously minimize the rms spot size on axis and at very small field angles. Since coma is the dominant geometrical aberration near the optical axis for telescopes without spherical aberration, this was an attempt to correct both spherical aberration and coma. A quasiaplanatic hyperboloid-hyperboloid design was achieved.

A comparison of the geometrical performance of the above two designs is illustrated in Fig. 7. At large field angles there is only modest improvement, since both designs suffer from the same field curvature and approximately the same astigmatism. From the inset we see that spherical aberration, though negligible, is not identically zero. However, coma has been substantially corrected, as evidenced by the small initial slope in the rms spot size versus field angle. These results are consistent with Nariai's attempt to correct coma with a hyperboloid-hyperboloid design. ${ }^{14,15}$ He was also able to substantially reduce, but not eliminate, coma.

If we consider only geometrical aberrations due to residual design errors, the percentage improvement achieved in optical performance by going from the classical Wolter type I design to this aplanatic hyperboloid-hyperboloid design is significant, as illustrated in Fig. 8. A maximum reduction in rms image size of $42 \%$ is achieved at a field angle of 1.3 arcmin.

The percentage improvement in the geometrical performance of a hyperboloid-hyperboloid aplanatic design over the classical paraboloid-hyperboloid Wolter type I telescope is much lower than that realized by the normalincidence Cassegrain telescopes shown in Fig. 2 and 3. Furthermore, it will be reduced even more when we perform a systems engineering analysis and consider image degradation mechanisms other than residual design errors.

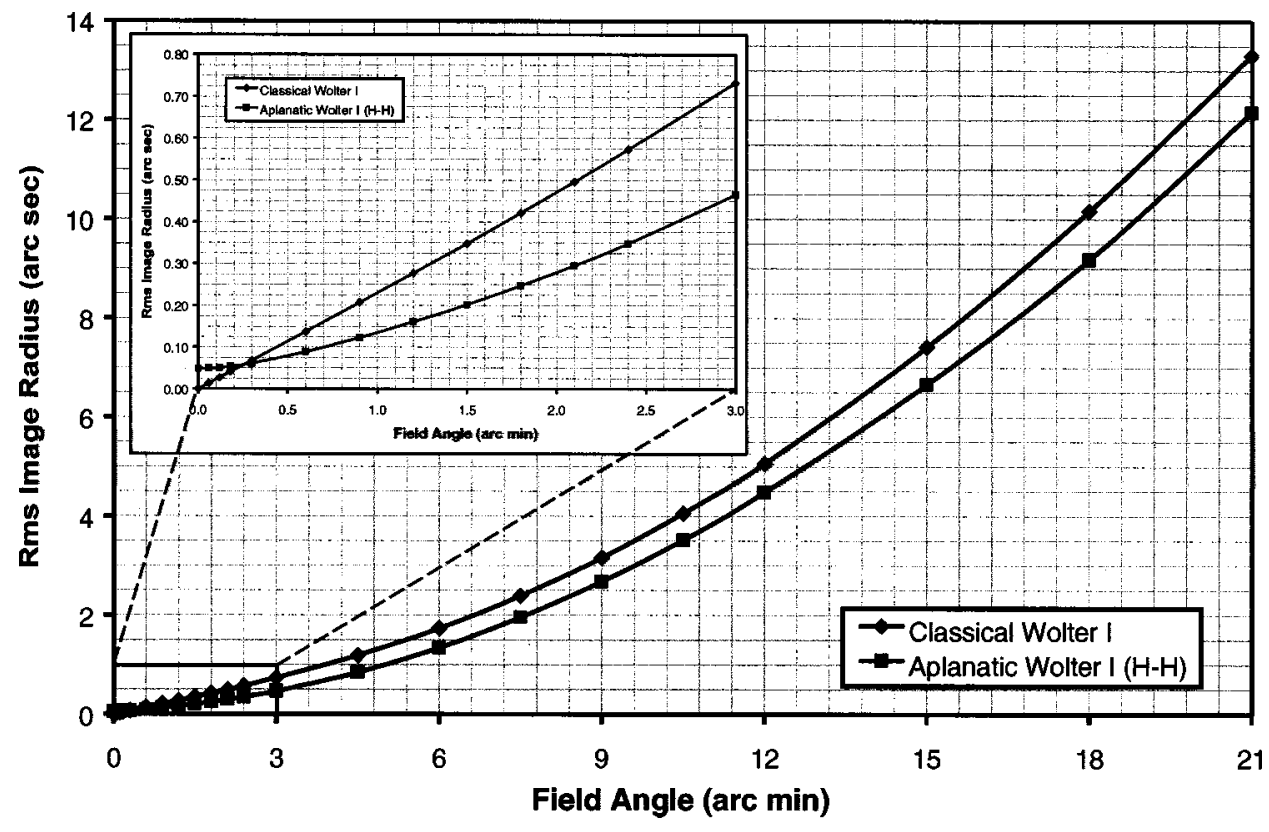

Fig. 7 Rms image radius plotted as a function of field angle for both a classical Wolter type I grazing incidence $\mathrm{x}$-ray telescope and its aplanatic hyperboloid-hyperboloid counterpart. 


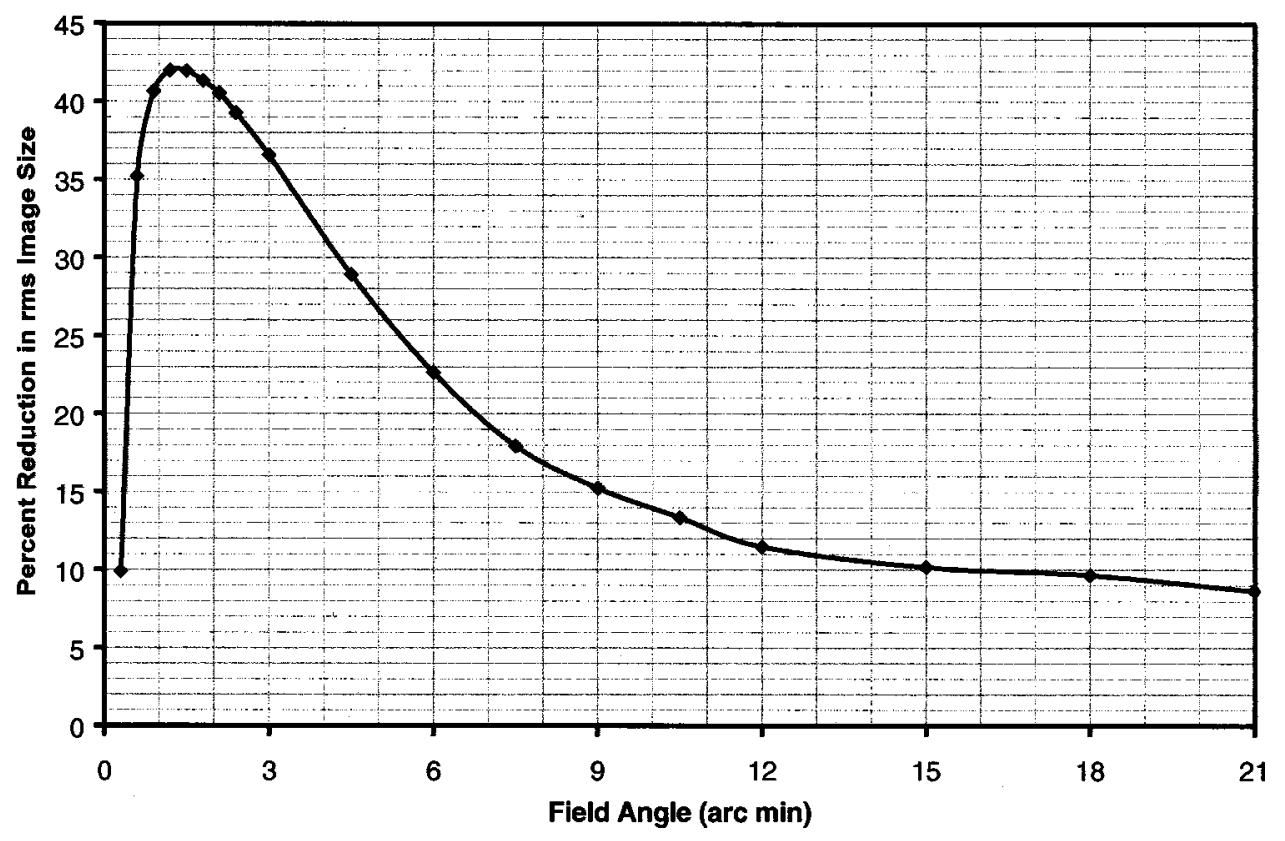

Fig. 8 Percentage improvement in the geometrical performance of an aplanatic hyperboloidhyperboloid variation of a classical Wolter type I grazing incidence x-ray telescope design.

\section{Systems Engineering Analysis and Performance Comparison}

A complete systems engineering analysis of this aplanatic Wolter Type I x-ray telescope requires that we look beyond the geometrical aberrations and include the effects of aperture diffraction, surface scattering from residual optical fabrication errors, assembly and alignment errors, metrology errors, etc. In this section we perform a detailed analysis of aperture diffraction and surface scattering phenomena for the SXI design parameters, then add the effects of all other potential error sources appearing in the SXI error budget tree as a single contribution to the final rms image core diameter.

\subsection{Image Degradation Due to Aperture Diffraction in Wolter Type I Telescopes}

The irradiance distribution of an aberration-free image formed by an annular aperture with a linear obscuration ratio $\epsilon$ is given by the well-known expression

$I(x)=\frac{1}{\left(1-\epsilon^{2}\right)^{2}}\left[\frac{2 J_{1}(x)}{x}-\epsilon^{2} \frac{2 J_{1}(\epsilon x)}{\epsilon x}\right]^{2}$,

where $x$ is a normalized (dimensionless) radius in the focal plane,

$x=\frac{\pi r}{\lambda f / D}$.

In 1974 , Tschunko ${ }^{26}$ showed that, for large obscuration ratios, squaring the sum of the two terms in Fig. 7 results in a dominant interference effect that produces an irradiance distribution made up of ring groups. For $\epsilon>0.8$, the number of rings in each ring group is given by $n=\frac{2}{1-\epsilon}$,

and the first ring group contains $90.28 \%$ of the point image flux.

Harvey $^{27}$ pointed out that for a Wolter type I x-ray telescope with an obscuration ratio $\epsilon=0.98$, there are 100 rings per ring group, and the central lobe of the diffractionlimited point spread function (PSF) contains only a very small fraction of the total energy in the PSF. It is thus the first ring group, not the central lobe of the diffraction pattern, that provides a meaningful measure of image size or resolution. The angular radius of the image is thus given by

$\theta_{0}=\frac{2 \lambda}{D(1-\epsilon)}$,

which is almost two orders of magnitude larger than the Airy disk of an unobscured circular aperture of the same diameter. Thus diffraction effects are not necessarily negligible, since the effects of the high obscuration ratios inherent in grazing incidence optics offsets the effects of the very short $\mathrm{x}$-ray wavelengths.

However, since the diffraction-limited PSF must be convolved with the geometrical PSF, which must in turn be convolved with the scattering function and the cumulative effects of all the other miscellaneous error sources, we have developed a semiempirical expression for the diffractionlimited irradiance distribution in the focal plane that does not require the high sampling density and associated computational problems encountered when performing these convolutions numerically. That empirical expression is given by 


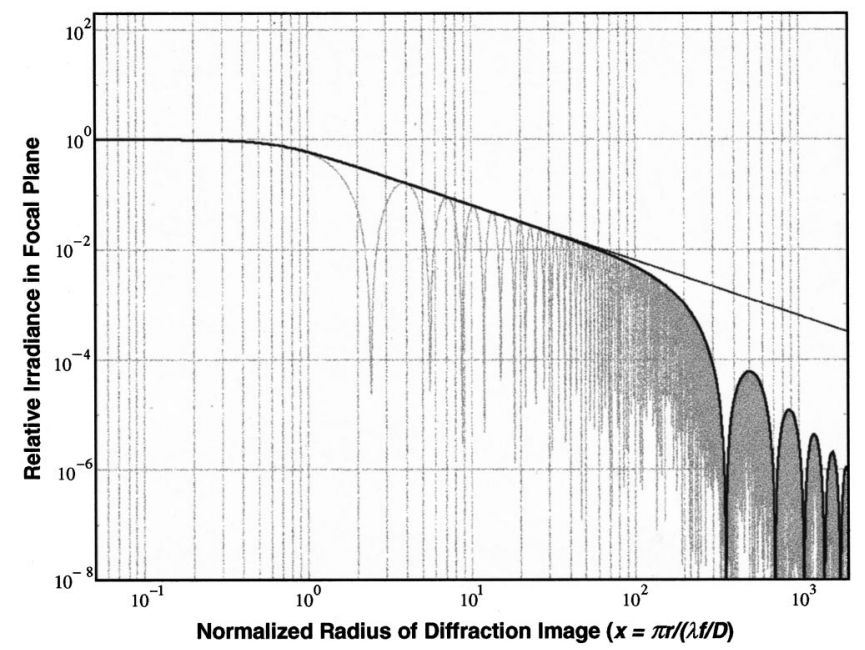

Fig. 9 Radial profile of the semi empirical expression used to approximate the diffraction-limited PSF.

$I(x)=a\left(\left|\frac{x}{0.643}\right|^{3}+1\right)^{-1 / 3}\left\{\frac{\sin [x(1-\epsilon) / 2]}{x(1-\epsilon) / 2}\right\}^{2}$.

This function does not exhibit the high-spatial-frequency diffractions rings, which will be smoothed out upon convolution with another function; however, it does accurately map the energy distribution among the ring groups as shown in Fig. 9. The constant $a$ is chosen to normalize the volume under the function to unity.

Equation (7) thus describes the diffraction-limited PSF of a Wolter type I x-ray telescope; however, for computational reasons Eq. (11) is used when calculating the system PSF as degraded by all system error sources. A threedimensional plot of this approximation to the diffractionlimited PSF for the SXI telescope $(\epsilon=0.9825)$ is presented below.

\subsection{Image Degradation Due to Surface Scattering in Wolter Type I Telescopes}

Since Ehrenberg's ${ }^{4}$ estimation of surface scattering in 1949 , the theory of wavefront perturbation and propagation has evolved to predict with accuracy the scattered intensity distributions from most optical surfaces. The current standard for surface scattering theory is Beckmann and Spizzichino' ${ }^{28}$ text; however, one encounters difficulty in extracting intuitive conclusions from it. Other useful references dealing with surface scattering for general optical applications include Introduction to Surface Roughness and Scattering by J. M. Bennett and L. Mattsson, ${ }^{29}$ Optical Scattering, Measurement and Analysis by John C. Stover, ${ }^{30}$ and a myriad of excellent papers published over the years by Eugene Church. ${ }^{31-35}$

The simplest and most intuitive way to approach statistical surface scattering is by way of a linear systems paradigm, which is an approach integrable with that of aperture diffraction and aberration theories. Harvey ${ }^{36-40}$ has shown that surface scattering phenomena can indeed be formulated through a linear systems treatment of scalar diffraction theory. The resulting surface transfer function is determined from the relevant surface statistics, viz., the surface

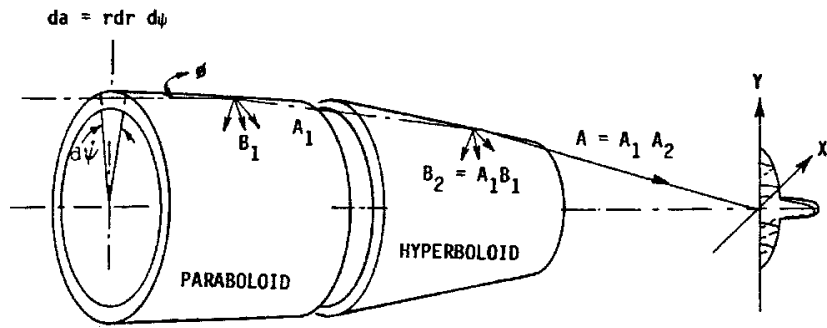

Fig. 10 Scattering geometry and degraded PSF for a typical Wolter type I telescope.

autocovariance (ACV) function or its Fourier transform, the surface power spectral density (PSD) function. Furthermore, this treatment is extendable to rough surfaces at extreme grazing angles, unlike its rigorous electromagnetic counterpart, which frequently employs perturbation techniques that restrict it to the "smooth" surface regime.

However, for the purposes of this paper, a practical implementation of Beckmann scattering theory in the computer program EEGRAZPC (Glenn et al. ${ }^{41}$ ) was utilized. The imaging characteristics of a Wolter type I x-ray telescope can be appreciated by considering the irradiance distribution produced in the focal plane from a single differential azimuthal section, or barrel stave, of the rotationally symmetric grazing incidence two-mirror system illustrated in Fig. 10. This irradiance distribution consists of an image core and an elongated scattering function as shown schematically in Fig. 11(a). The elongated scattering function results from the foreshortening effect of the axial projection of surface height variations onto the reflected wavefront. ${ }^{37}$ Integration of the differential azimuthal aperture element from zero to $2 \pi$ merely results in the superposition of many rotated elongated one-dimensional scattering functions as depicted in Fig. 11(b). Clearly, the fractional encircled energy of the resulting composite rotationally symmetric PSF (superposition of rotated elongated scattering functions) is the same as the fractional encircled energy of the single elongated scattering function shown in Fig. 11(a). EEGRAZ utilizes this fact to reduce computationally intensive 2-D fast Fourier transform (FFT) calculations to the much simpler and less time-consuming 1-D calculations. EEGRAZ was utilized extensively on NASA's AXAF pro-

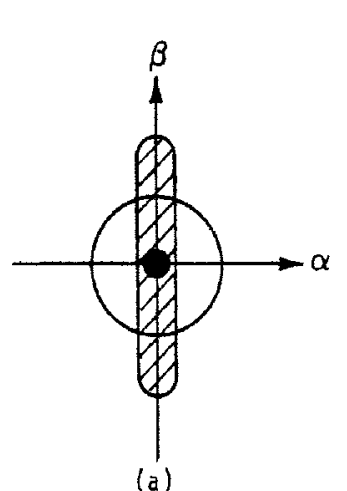

(a)

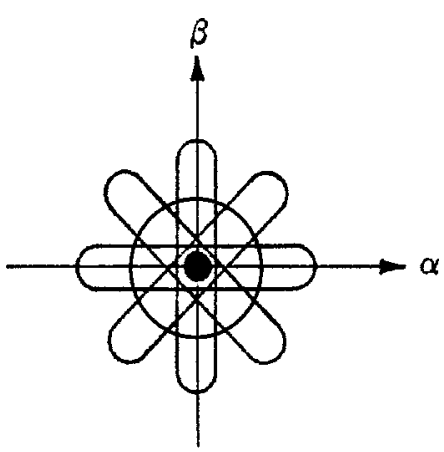

(b)
Fig. 11 (a) Elongated scattering function due to illumination of a single barrel stave at $\psi=90$ deg. (b) Rotationally symmetric scattering function when an entire annular aperture is illuminated. 
Thompson and Harvey: Systems engineering analysis ...

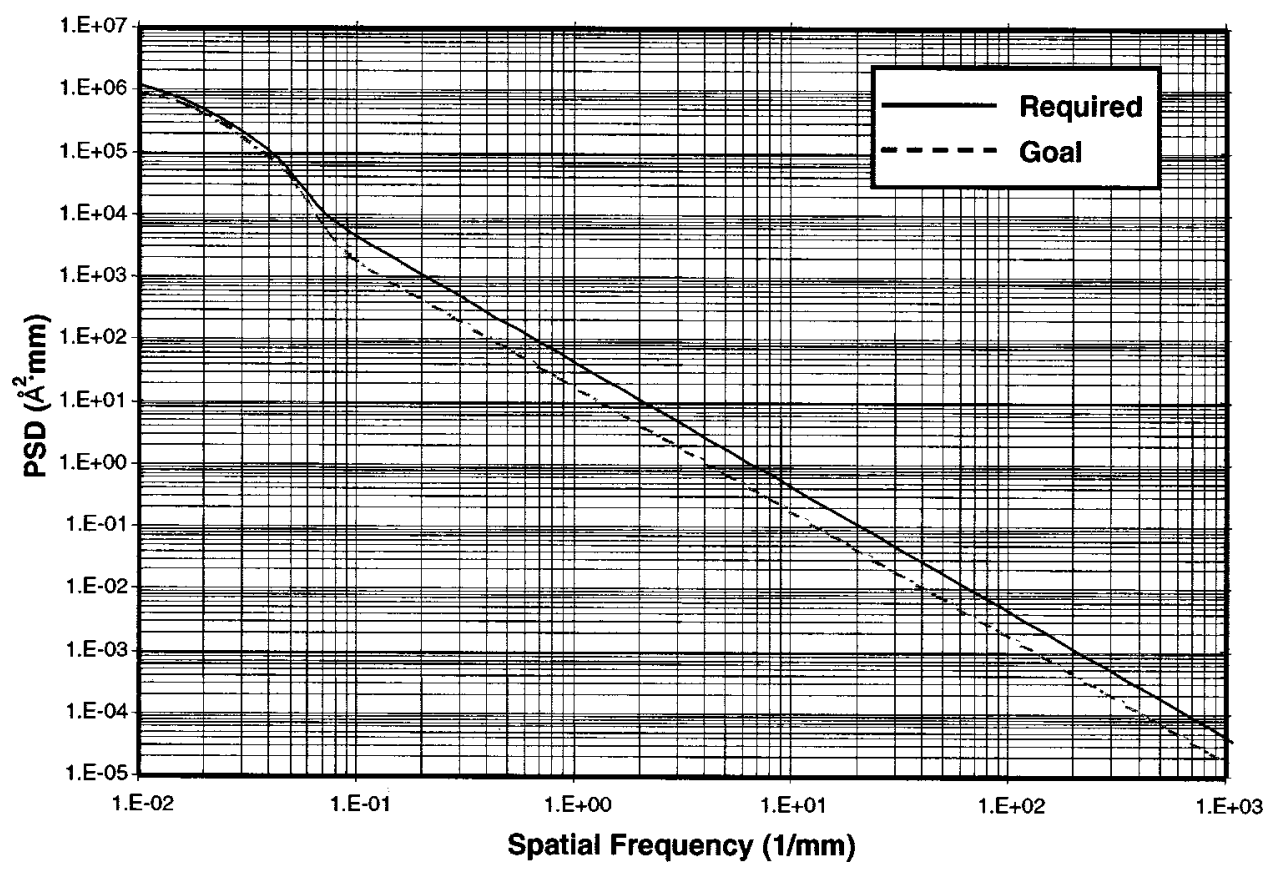

Fig. 12 Optical fabrication tolerances specified for the SXI mirrors expressed as required and goal PSDs.

gram and has been experimentally validated for Wolter type I x-ray telescopes operating at small field angles, i.e., for small geometrical aberrations.

Information concerning the relevant surface statistics (residual optical fabrication errors) must be provided as input to the EEGRAZ code in the form of either an average axial surface ACV function or an average axial surface PSD function. Other necessary input includes the average grazing angle of the Wolter type I telescope and the wavelengths or X-ray energies for which the analysis is desired.

For SXI, preliminary analysis showed that an average axial ACV function synthesized by summing two Gaussian functions and one inverse exponential function should satisfy the top-level image quality requirement. ${ }^{42}$ Each of the three functional components in the ACV is determined by an rms roughness $\sigma$ and a correlation length $L$ :

$$
\begin{aligned}
\operatorname{ACV}(x)= & \sigma_{1}^{2} \exp \left[-\left(x / L_{1}\right)^{2}\right]+\sigma_{2}^{2} \exp \left[-\left(x / L_{2}\right)^{2}\right] \\
& +\sigma_{3}^{2} \exp \left(-\left|x / L_{3}\right|\right)
\end{aligned}
$$

where

$\sigma_{1}=\sigma_{2}=100 \AA, \quad \sigma_{3}=140 \AA \quad$ (required)

or $90 \AA$ (goal),

$L_{1}=10 \mathrm{~mm}, L_{2}=20 \mathrm{~mm}$, and $L_{3}=45 \mathrm{~mm}$.

The surface PSD (Fig. 12) is given by the Fourier transform of the ACV function. Note that since Fig. 12 is a single-sided representation of the full PSD, Eq. (13) must be multiplied by two in order to generate the exact curves displayed in Fig. 12:

$$
\begin{aligned}
\operatorname{PSD}(f)= & \sigma_{1}^{2} L_{1} \sqrt{\pi} \exp \left[-\left(\pi L_{1} f\right)^{2}\right]+\sigma_{2}^{2} L_{2} \sqrt{\pi} \\
& \times \exp \left[-\left(\pi L_{2} f\right)^{2}\right]+\frac{2 \sigma_{3}^{2} L_{3}}{1+\left(2 \pi L_{3} f\right)^{2}} .
\end{aligned}
$$

This surface PSD is thus a convenient specification for residual optical fabrication errors, and becomes a contractional requirement for the mirror manufacturer. Two or three different metrology instruments must typically be used to span the range of spatial frequencies from conventional figure errors to microroughness or finish errors.

Providing this one-dimensional surface PSD as input to EEGRAZ allows us to calculate the one-dimensional scattering function that would result from illumination of a single barrel stave of the Wolter type I grazing incidence telescope. That one-dimensional scattering function is then converted to a rotationally symmetric two-dimensional scattering function that can be convolved with the geometrical PSF, the diffraction-limited PSF, and the image core that results from all of the other potential error sources in the error budget tree.

\subsection{Total Image Degradation Including All Other Residual Errors}

The optical system PSF (or aerial image, since we are ignoring detector effects), as degraded by a variety of independent and uncorrelated error sources, can be approximated by the convolution of the individual PSFs associated with the respective error sources. ${ }^{37}$ The effect of all miscellaneous error sources in the error budget tree on the onedimensional image core diameter (produced by a single barrel stave) can be root-sum-squared (rss'd) as illustrated in Fig. 13. These error budget allocations are those of the mirror manufacturer for the SXI grazing incidence $\mathrm{x}$-ray 


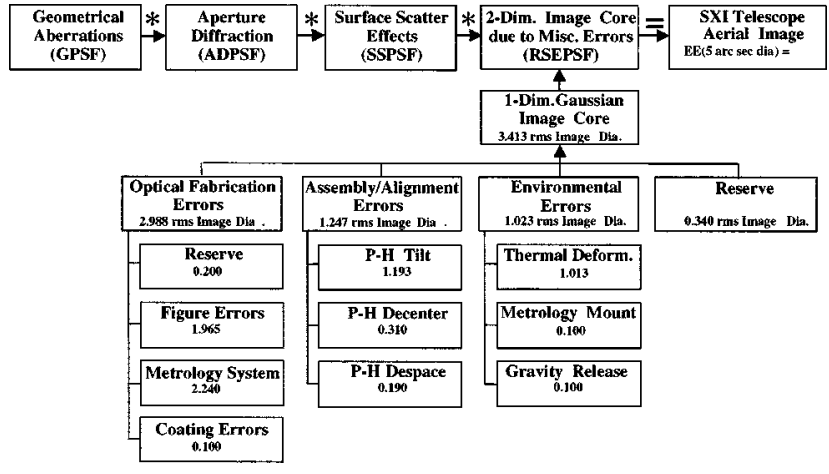

Fig. 13 Error budget tree for manufacturing the SXI mirrors ( $*$ is a symbol for a two-dimensional convolution operation).

telescope. ${ }^{42}$ This one-dimensional image core can be assumed to have a Gaussian shape, since the central limit theorem of Fourier transform theory states that "the convolution of $n$ uncorrelated functions tends toward a gaussian as $n$ tends toward infinity." ${ }^{43}$ This one-dimensional Gaussian function is then converted to a two-dimensional image core, which is then convolved with the result of convolving the three dominant image degradation mechanisms discussed earlier.

A three-dimensional plot of the PSF produced by each of the three dominant image degradation mechanisms-(1) aperture diffraction, (2) geometrical aberrations, and (3) surface scattering due to residual optical fabrication errors-is given in Fig. 14 along with a similar plot of the two-dimensional image core due to miscellaneous residual

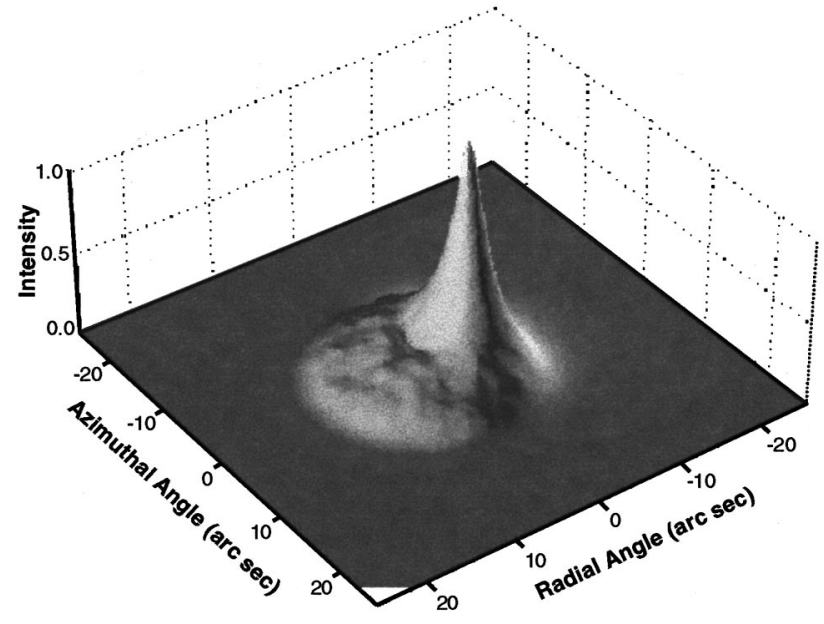

Fig. 15 Illustration of the system PSF (or aerial image) of a Wolter type I grazing incidence $\mathrm{X}$-ray telescope with the SXI mirror specifications at a field angle of 15 arcmin and a wavelength of $44.7 \AA$.

errors. The geometrical aberrations are a strong function of field angle, and both diffraction effects and surface scattering are strong functions of wavelength; however, we have chosen a field angle of 15 arcmin and a wavelength of 44.7 $\AA$ for the examples displayed in Fig. 14 .

The convolution these four functions provides the predicted SXI aerial image. This system PSF is illustrated in Fig. 15. Once we have the optical system's PSF, we can calculate a variety of commonly used image quality criteria. Choosing the half-power radius (HPR) versus field

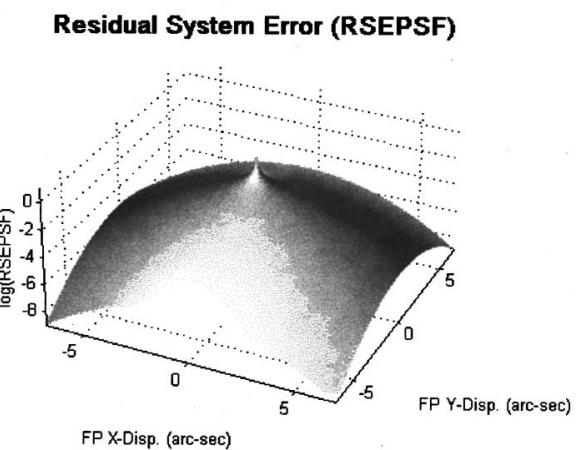

\section{Geometrical Spot Density (GPSF)}

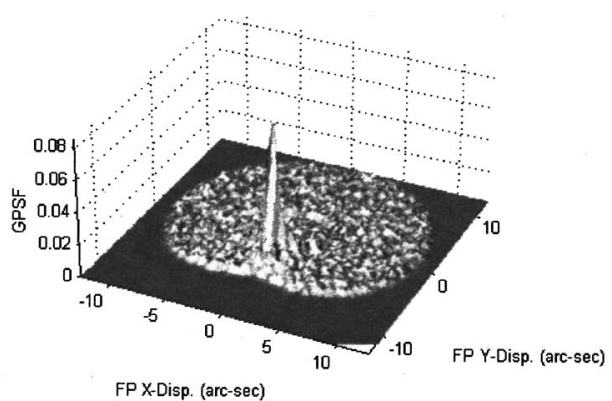

Fig. 14 Illustration of the individual image degradation mechanisms for a Wolter type I grazing incidence $x$-ray telescope with the SXI mirror specifications at a field angle of $15 \mathrm{arcmin}$ and a wavelength of $44.7 \AA$. 


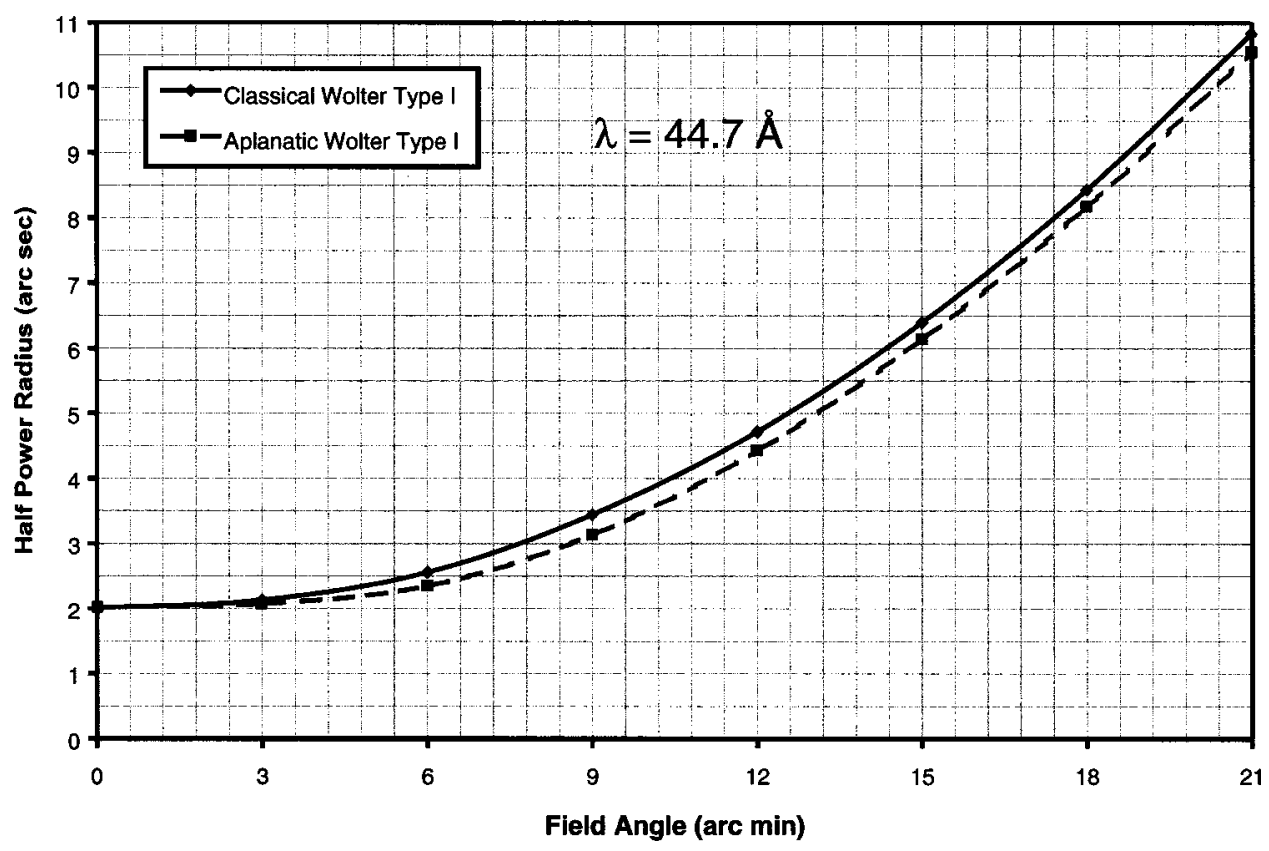

Fig. 16 Comparison of the HPR versus field angle for the classical Wolter type I design and an aplanatic hyperboloid-hyperboloid design $(\lambda=44.7 \AA)$ with the same first-order properties.

angle as a meaningful characterization of the performance of a grazing incidence $\mathrm{x}$-ray telescope, we performed the above calculations for several field angles and two different wavelengths for both the classical Wolter type I design and the aplanatic hyperboloid-hyperboloid design discussed earlier. A performance comparison of these two designs $(\lambda=44.7 \AA)$ is given in Fig. 16 .

Figure 17 shows the percentage reduction in HPR for two different $\mathrm{x}$-ray wavelengths when going from the classical Wolter type I design to the aplanatic hyperboloid- hyperboloid design discussed above. The previous curve showing the percentage reduction in rms image radius when considering residual design errors only, and the corresponding improvement for the normal-incidence aplanatic Cassegrain operating at visible wavelengths, are also shown for comparison.

Note that there is less than a $10 \%$ reduction in the HPR on going to the aplanatic design. At the small field angles that yielded significant improvement in the geometrical performance, the system performance is limited by scatter-

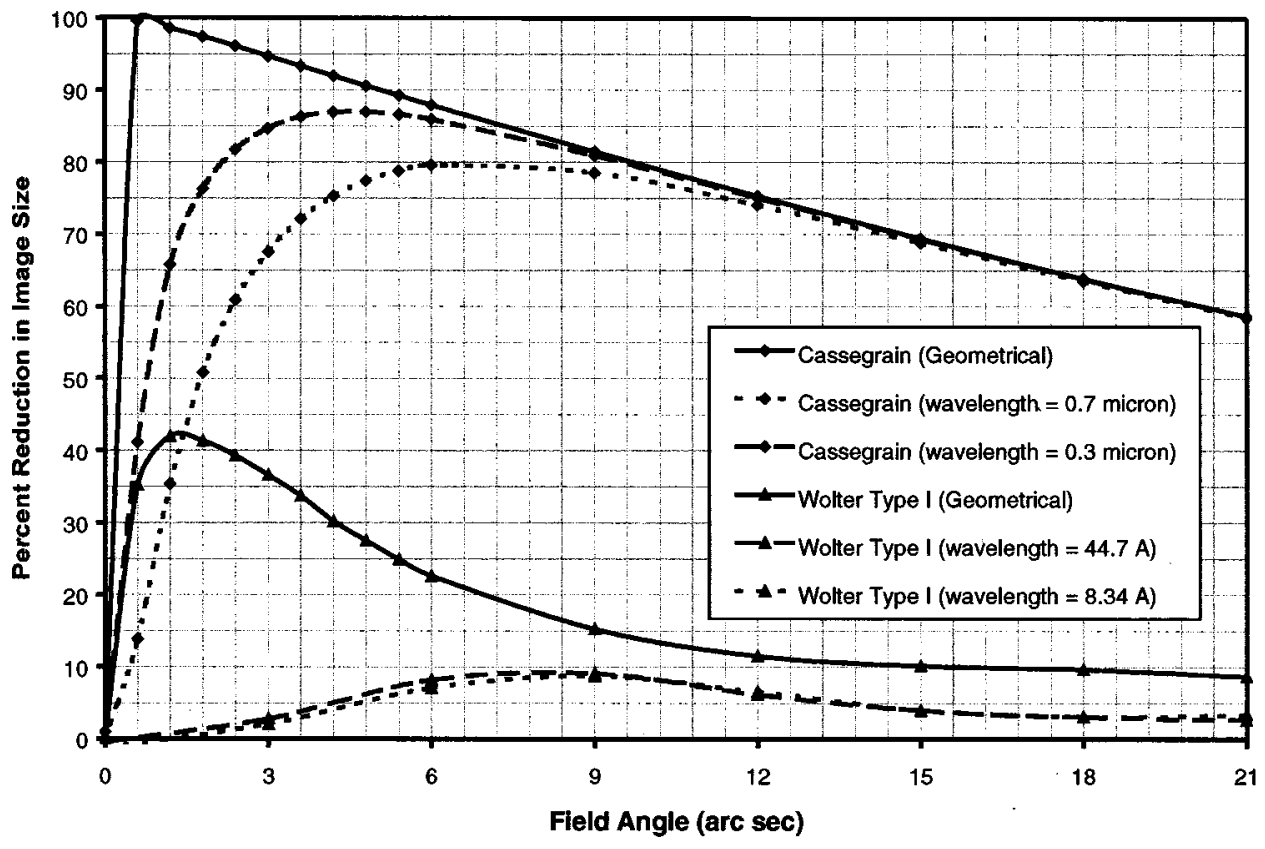

Fig. 17 There is only a very modest improvement $(<10 \%)$ in the system performance of an aplanatic Wolter type I x-ray telescope design. 
ing effects and other error sources. At large field angles, the image quality is limited by field curvature and astigmatism, which are about the same for both designs. Hence, there is little practical advantage to an aplanatic design for grazing incidence $\mathrm{x}$-ray telescopes.

\section{Summary and Conclusion}

A historical background and an overview of contemporary progress in the expanding field of $\mathrm{x}$-ray imaging and grazing incidence telescope design has been presented in this paper. This retrospective was done in order to draw a thread of reasoning, reaching as far back as 1672 , to the effect that telescope designs are only as good as can be practically implemented.

In Sec. 2, we reviewed the substantial improvement achieved by aplanatic Cassegrain (Ritchey-Chretein) telescope designs operating at visible wavelengths. This has led many researchers to expect similar performance improvements in aplanatic Wolter type I designs.

We then reviewed the fundamentals of grazing incidence Wolter type I telescope designs, and a comparison of the geometrical performance of a particular Wolter type I design and an aplanatic hyperboloid-hyperboloid variation of it.

Finally, we performed an extensive systems engineering analysis of the above two telescopes, including not only the effects of geometrical aberrations, but also the effects of diffraction, scattering, misalignments, etc. In particular, we showed that the diffraction effects of highly obscured annular apertures largely offsets the effect of very short wavelengths; hence, diffraction effects are not always negligible in x-ray imaging systems. Surface scattering effects from residual optical fabrication errors were also analyzed in detail. At very short $\mathrm{x}$-ray wavelengths, these scattering effects, even for our smoothest surfaces, frequently dominate geometrical aberrations for small field angles. Finally, we included the effects of all other potential error sources in the mirror manufacturer's error budget tree.

The results of this systems engineering analysis indicates that an aplanatic Wolter type I design will not significantly improve the image quality at $\mathrm{x}$-ray wavelengths. Surface scattering effects (and other system errors) dominate the effects of coma for small field angles, and field curvature and astigmatism dominate the effects of coma at large field angles. Figure 17 provides a dramatic comparison of the performance improvement of an aplanatic Wolter type I grazing incidence $\mathrm{x}$-ray telescope with that of a normal-incidence Ritchey-Chretien design operating at visible wavelengths.

\section{Acknowledgments}

We would like to thank Lockheed-Martin Solar and Astrophysics Laboratory (LMSAL) for their encouragement and informative participation in this research, as well as Lockheed-Martin Missiles and Space (LMMS) for the financial support crucial to work of this kind. We would also mention that Raytheon Optical Systems, Inc. (ROSI) have been of tremendous help by allowing us insight into their knowledge of x-ray and Wolter type I optical systems engineering, and by providing us with the current version of EEGRAZPC.

\section{References}

1. H. C. King, The History of the Telescope, Dover Publications, New York (1979)

2. E. Hecht, Optics, 2nd ed., Addison-Wesley Publishing Company, Reading, MA (1987).

3. R. N. Wilson, Reflecting Telescope Optics I, Basic Design Theory and Its Historical Development, Springer (1996).

4. B. Aschenbach, "X-ray telescopes," Rep. Prog. Phys. 48, 579-629 (1985).

5. P. Kirkpatrick and A. V. Baez, "Formation of optical images by x-rays," J. Opt. Soc. Am. 38, 776 (1948).

6. H. Wolter, "Mirror systems with glancing incidence on image producing optics for x-rays," Ann. Phys. (N.Y.) 10, 94 (1952).

7. H. Wolter, "Generalized Schwarzschild mirror systems with glancing incidence as optics for x-rays," Ann. Phys. (N.Y.) 10, 286 (1952).

8. L. P. Van Speybroeck and R. C. Chase, "Design parameters of paraboloid-hyperboloid telescopes for x-ray astronomy," Appl. Opt. 11, 440-445 (1972)

9. R. C. Chase and L. P. Van Speybroeck, "Wolter-Schwarzschild telescopes for x-ray astronomy," Appl. Opt. 12, 1042-1044 (1973).

10. W. Werner, "Imaging properties of Wolter I type x-ray telescopes," Appl. Opt. 16, $764-773$ (1977).

11. C. E. Winkler and D. Korsch, "Primary aberrations for grazing incidence," Appl. Opt. 16, 2464-2469 (1977).

12. W. Cash, D. L. Sheeley, and J. H. Underwood, Proc. SPIE 184, 228 (1979).

13. D. Korsch, Reflective Optics, Chap. 11, pp. 282-284, Academic Press, Boston (1991).

14. K. Nariai, "Geometrical aberrations of a generalized Wolter type I telescope," Appl. Opt. 26, 4428-4432 (1987).

15. K. Nariai, "Geometrical aberrations of a generalized Wolter type I telescope 2: analytical study," Appl. Opt. 27, 345-350 (1988).

16. T. T. Saha, "Transverse ray aberrations for paraboloid-hyperboloid telescopes," Appl. Opt. 24, 1856-1863 (1985).

17. T. T. Saha, "Transverse ray aberrations of Wolter type I telescopes," Proc. SPIE 640, 10-19 (1986).

18. T. T. Saha, "General surface equations for glancing incidence telescopes,” Appl. Opt. 26, 658-663 (1987).

19. T. T. Saha, "Aberrations for grazing incidence telescopes," Appl. Opt. 27, 1492-1498 (1988)

20. P. Bornman, D. Speich, J. Hirman, V. Pizzo, R. Grubb, C. Balch, and G. Heckman, "GOES solar x-ray imager: overview and operational goals," in GOES-8 and Beyond, E. R. Washwell, Ed., Proc. SPIE 2812, 309-319 (1996).

21. J. E. Harvey, K. L. Lewotsky, and A. Kotha, "Performance predictions of a Schwarzschild imaging microscope for soft x-ray applications," Opt. Eng. 35, 2423-2436 (1996).

22. H. H. Hopkins, Wave Theory of Aberrations, Clarendon Press, Oxford (1950).

23. V. N. Majahan, Optical Imaging and Aberrations, SPIE Optical Engineering Press (1998)

24. http://www.focus-software.com/zemax/index.html

25. R. J. Noll, P. E. Glenn, and J. Osantowski, "Optical surface analysis code (OSAC)," Proc. SPIE 362, 78 (1982).

26. H. F. A. Tschunko, "Imaging performance of annular apertures," Appl. Opt. 13, 1820-1823 (1974)

27. J. E. Harvey, "Diffraction effects in grazing incidence $\mathrm{x}$-ray telescopes,'” J. X-Ray Sci. Technol. 3, 68-76 (1991).

28. P. Beckman and A. Spizzichino, The Scattering of Electromagnetic Waves from Rough Surfaces, Pergamon Press, New York (1963).

29. J. M. Bennett and L. Mattsson, Introduction to Surface Roughness and Scattering, Opt. Soc. Am., Washington (1989)

30. J. C. Stover, Optical Scattering, Measurement and Analysis, McGrawHill, New York (1990).

31. E. L. Church, "The role of surface topography in x-ray scattering," Proc. SPIE 184, 196 (1979)

32. E. L. Church, "Small-angle scattering from smooth surfaces," (abstract only) J. Opt. Soc. Am. 70, 1592 (1980)

33. E. L. Church, "Interpretation of high-resolution x-ray scattering measurements," Proc. SPIE 257, 254 (1980).

34. E. L. Church, "Fractal surface finish," Appl. Opt. 27, 1518-1526 (1988).

35. E. L. Church and P. Z. Takacs, "Specification of the surface and finish of optical elements in terms of system performance," Proc SPIE 1791, (1992)

36. J. E. Harvey, "Light-scattering characteristics of optical surfaces," $\mathrm{PhD}$ Dissertation, The University of Arizona (1976).

37. J. E. Harvey, E. C. Moran, and W. P. Zmek, "Transfer function characterization of grazing incidence optical systems," Appl. Opt. 27, $1527-1533$ (1988)

38. J. E. Harvey, "Surface scatter phenomena: a linear, shift-invariant process," in Scatter from Optical Components, J. C. Stover, Ed., Proc. SPIE 1165, 87-99 (1989).

39. J. E. Harvey, "Potential pitfalls in the design of x-ray/EUV optics," 
in Lens Design: Critical Reviews of Optical Science and Technology, W. J. Smith, Ed., Proc. SPIE CR41, (1992).

40. J. E. Harvey, "Scattering effects in x-ray imaging systems," Proc. SPIE 2515, 32 (1995).

41. P. Glenn, P. Reid, A. Slomba, L. P. Van Speybroeck, "Performance prediction of AXAF technology mirror assembly using measured mirror surface errors," Appl. Opt. 27, 1539-1543 (1988).

42. Raytheon Optical Systems Inc., SXI Mirror Assembly Critical Design Review, Document No. VG P23-0021 (1998).

43. J. D. Gaskill, Linear Systems, Fourier Transforms, and Optics, John Wiley \& Sons, New York (1978).

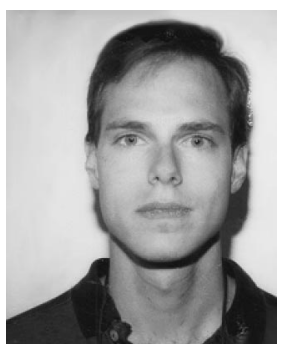

Patrick L. Thompson received his BS and MS in applied physics (with certificate in optics) from the Georgia Institute of Technology under Dr. Donald O'Shea. He is currently working toward his $\mathrm{PhD}$ in Optical Sciences and Engineering at CREOL (University of Central Florida), where he is studying $\mathrm{x}$-ray mirror design and performance for space science applications. Mr. Thompson has been employed by HughesDanbury Optical Systems (AXAF program), Lockheed-Martin Aeronautical Systems Company (F-22, C-130 pro- grams), Advanced Optical Systems Inc. (optical computing), and the Georgia Tech Research Institute (microwave antenna and radar cross section analysis). He is a member of SPIE, OSA, and AIAA.

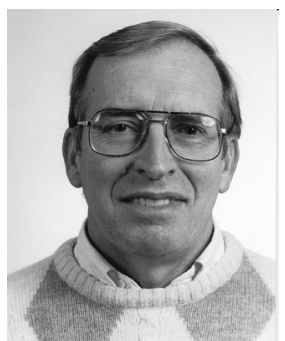

James E. Harvey received his PhD in optical sciences from the University of Arizona in 1976 . He is currently an associate professor in the School of Optics at the University of Central Florida and a Senior Staff Member at the Center for Research and Education in Optics and Lasers (CREOL). Dr. Harvey is credited with over one hundred publications and presentations in the areas of diffraction theory, surface scattering phenomena, adaptive optics, wavefront sensing, beam sampling technology, optical properties of materials, and x-ray/EUV imaging systems. He is a member of the OSA and a Fellow of SPIE. 